\title{
Clinical Diagnosis of Invasive Pulmonary Aspergillosis in a Non-Neutropenic Critically Ill Patient
}

\author{
Jean Turc MD, Antoine Lamblin MD, Thierry Vitry MD, Frédéric Carémil MD, \\ Christophe Lions MD, Eric Dardare MD, and Olivier Bylicki MD
}

\section{Introduction}

Invasive pulmonary aspergillosis (IPA) is a life-threatening fungal infection that predominantly affects severely immunocompromised patients, particularly those with prolonged neutropenia or organ transplantation. ${ }^{1}$ Definitions have been developed that facilitate the diagnosis of IPA in immunocompromised patients with cancer or hematologic malignancy. ${ }^{2}$ Subsequent publications explored IPA in nonimmunocompromised patients in ICUs. ${ }^{3,4}$ In this specific setting, diagnosis of IPA is challenging for several reasons: it is a relatively uncommon condition, clinical presentation may be unspecific and mimic ventilator-associated pneumonia, and specific radiological and microbiological findings may be delayed. To highlight the difficulties of IPA diagnosis in the ICU, we present the case of an 82-y-old ICU patient without immunosuppression affected by possible IPA.

\section{Case Summary}

An 82-y-old woman was referred to the ICU at Desgenettes Military Hospital (Lyon, France) for septic shock and acute kidney injury due to acute peritonitis. Before the onset of the symptoms, she was in good health without any chronic medication, and her past medical history was significant only for hypertension. She was a nonsmoker. She underwent surgery, and supportive care was initiated with broad spectrum antibiotics, vasopressor support, mechan-

Drs Turc, Lamblin, Carémil, Lions, and Dardare are affiliated with the Department of Anesthesiology and Intensive Care; Dr Vitry is affiliated with the Medical Imaging Department; and Dr Bylicki is affiliated with the Respiratory Department, Desgenettes Military Hospital, Lyon, France.

The authors have disclosed no conflicts of interest.

Correspondence: Jean Turc MD, Department of Anesthesiology and Intensive Care, Desgenettes Military Hospital, 108 Pinel Boulevard, Lyon 69008, France. E-mail: jeanturc@free.fr.

DOI: $10.4187 /$ respcare.02579 ical ventilation, and intravenous hydrocortisone $(200 \mathrm{mg} / \mathrm{d})$ treatment. The initial evaluation was favorable, except for delayed awakening. At day 10, her condition worsened with fever and purulent sputum, suggesting ventilator-associated pneumonia.

On physical examination, her temperature was $38.5^{\circ} \mathrm{C}$, heart rate was 89 beats/min (rhythmic), blood pressure was $103 / 53 \mathrm{~mm} \mathrm{Hg}$, breathing frequency was 24 breaths/min, and resting oxygen saturation was $92 \%$ on mechanical ventilation (continuous spontaneous ventilation, PEEP $5 \mathrm{~cm} \mathrm{H}_{2} \mathrm{O}$, pressure support $18 \mathrm{~cm} \mathrm{H}_{2} \mathrm{O}$, and $\mathrm{F}_{\mathrm{IO}_{2}} 0.30$ ). Examination of the chest revealed bilateral rhonchi. Neurological status was altered: coma without focal sign, attributed to delayed awakening. Cardiac and abdominal statuses were normal. Laboratory findings were as follows: elevated neutrophil count $(18,100$ cells $/ \mathrm{mL})$, hemoglobin $(9.2 \mathrm{~g} / \mathrm{dL})$, platelets $(224,000$ cells $/ \mathrm{mL})$, elevated C-reactive protein $(110 \mathrm{mg} / \mathrm{L})$, and procalcitonin $(1.05 \mathrm{ng} / \mathrm{L})$. Arterial blood gas analyses showed respiratory alkalosis $\left(\mathrm{pH} 7.48, \mathrm{P}_{\mathrm{aCO}_{2}} 28 \mathrm{~mm} \mathrm{Hg}\right)$ and normoxia $\left(\mathrm{P}_{\mathrm{aO}_{2}} 95 \mathrm{~mm} \mathrm{Hg}\right)$ on mechanical ventilation.

Chest $\mathrm{x}$-ray showed only limited right pleural effusion. Cardiac ultrasound demonstrated normal right and left ventricular filling pressure. A CT scan was performed and revealed the presence of three parenchymal cavitary lesions (Fig. 1). These lesions were surrounded by a zone of lower attenuation with ground-glass opacities, forming the halo sign. An air crescent sign was also present, with crescentic radiolucencies surrounding an intracavitary nodular area of consolidation. Cavities were in continuity with bronchi. Interestingly, these findings were absent on a previous CT scan performed at day 1 (Fig. 2).

Fiberoptic bronchoscopy revealed purulent sputum and mucosal inflammation, but no alveolar hemorrhage. Direct examination of two bronchoalveolar lavage (BAL) fluid samples gave negative results (nonfungal hyphae, nonhyphae Candida, nonmycobacterial infection), whereas both cultures were found to be positive for Aspergillus fumigatus. Repeated serologic tests including galactomannan antigen assay and Aspergillus-specific antibodies were negative. Antigen detection or polymerase chain reaction in 


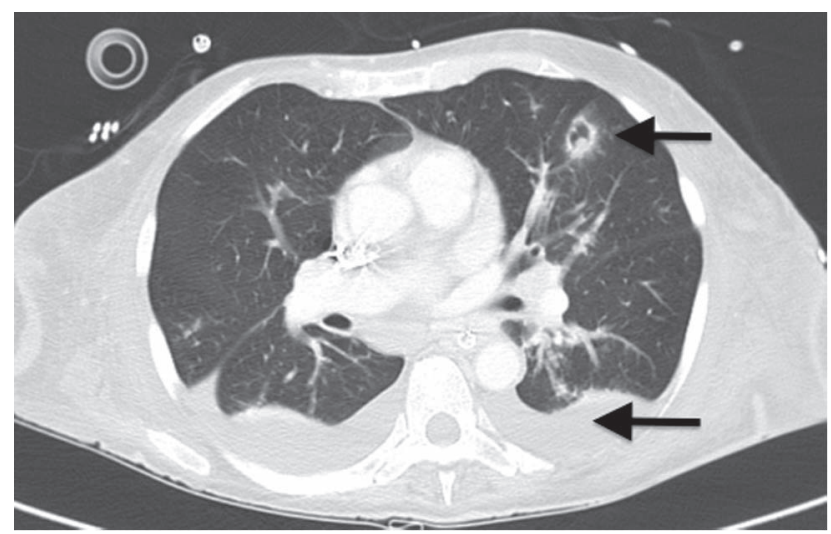

Fig. 1. Computed tomography scan of the chest performed at day 10 showing a centimetric cavity in the lingula, in continuity with the corresponding segmental bronchus (arrows). See the air crescent sign and perilesional ground-glass opacity. A minor bilateral pleural effusion is associated.

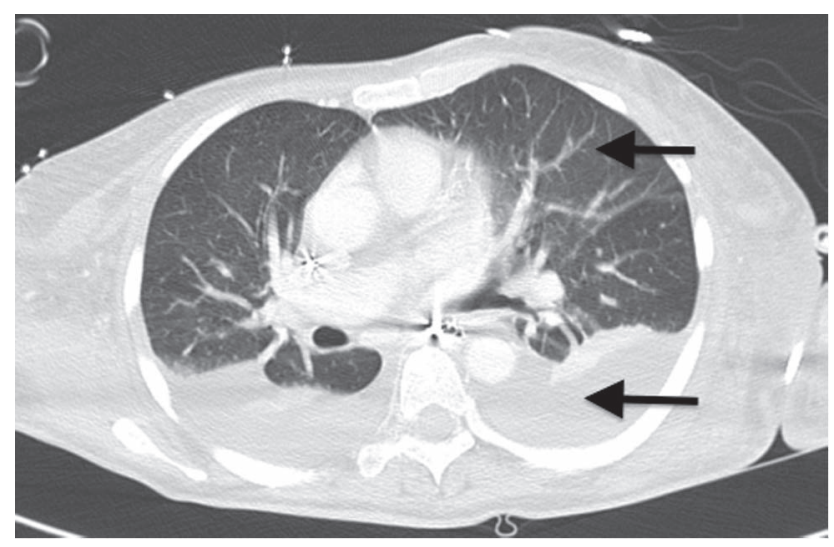

Fig. 2. Computed tomography (CT) scan of the chest performed at day 1 , showing aspecific bilateral pleural effusion. Note the absence of the cavity (arrows) observed in Figure 1 on this CT scan (which was performed earlier).

BAL fluid was not available at our center. The patient was managed with intravenous antifungal treatment (voriconazole at $6 \mathrm{mg} / \mathrm{kg} / 12 \mathrm{~h}$ the first day of treatment and $4 \mathrm{mg} /$ $\mathrm{kg} / 12 \mathrm{~h}$ on day 7) followed by voriconazole taken orally (400 mg/d). During treatment, the patient was stabilized, but she suffered from delayed awakening. A right eye deviation was subsequently observed. Brain imaging was consistent with a large right parieto-occipital ischemic stroke, leading to a limitation of treatment, and the patient eventually died.

\section{Discussion}

IPA predominantly affects severely immunocompromised patients, particularly those with prolonged neutropenia or organ transplantation: bone marrow, stem cell, or solid organ transplant recipients, as well as patients with chronic granulomatous disease. ${ }^{1,5}$ It has also emerged in the non-neutropenic patient and particularly in the ICU.3,4,6 IPA manifests as one of four major clinicopathological forms: acute bronchopneumonia, acute tracheobronchitis, angioinvasive aspergillosis, and chronic necrotizing aspergillosis. ${ }^{7,8}$ This case points out the aspecific presentation of invasive aspergillosis, mimicking ventilator-associated pneumonia in patients without specific risk factors in the ICU.

Aspergillus species have emerged as agents of nosocomial opportunistic infections in ICU patients. ${ }^{3}$ Risk factors for these infections are numerous and include sepsis-induced immunosuppression, prolonged systemic corticosteroid treatment, cirrhosis, COPD, and solid malignancy. ${ }^{1,2,4}$ Disease manifestations are usually less aggressive than the invasive disease that develops in neutropenic patients. As in the present case, clinical signs do not necessarily differ from those present with other causes of nosocomial pneumonia.

In 2008, a consensus group of the European Organization for Research and Treatment of Cancer/Invasive Fungal Infections Cooperative Group and the National Institute of Allergy and Infectious Diseases Mycoses Study Group (EORTC/MSG) published revised standard definitions of invasive fungal disease. ${ }^{2}$ The definitions assigned three levels of probability to the diagnosis of invasive fungal infection that develops in immunocompromised patients with cancer and in hematopoietic stem cell transplant recipients-namely, proven, probable, and possible invasive fungal infection. ${ }^{2}$ The definitions established a formal framework for defining invasive fungal infection with a variable certainty of diagnosis. Proven IPA required histopathologic or microbiologic documentation of infection from tissues obtained by biopsy or in culture samples from a normally sterile site. Probable IPA disease required (1) host factors, including a recent history of neutropenia, prolonged corticosteroid use, or treatment with $\mathrm{T}$ cell immunosuppressants; (2) clinical features, including the presence of a new infiltrate on a chest CT scan, such as dense lesions, air crescent signs, or a cavity; and (3) mycologic evidence. The category of possible IPA included those cases with sufficient clinical evidence consistent with IPA but for which there was no mycological support or no conventional host factor. In this case, despite the presence of mycological support, the absence of host factors leads to categorization as possible IPA.

Diagnosis remains challenging in this ICU population. Histopathologic evidence is the accepted standard, but biopsies are often not feasible in this population. ${ }^{9}$ Standard diagnosis is based on a number of arguments (clinical, radiological, biological, and microbiological). Standard diagnostic tools such as serum galactomannan antigen assay and Aspergillus-specific antibodies have been evaluated in neutropenic patients, but data in ICU patients are sparse. ${ }^{10-12}$ 
The clinical sensitivity of serum galactomannan is somewhat variable, within a range of $29-100 \% .^{12}$ Isolation of Aspergillus in BAL fluid may reflect either colonization or infection. ${ }^{13}$ Another disadvantage of culture is that it is relatively slow, relatively insensitive, and requires specialized expertise for species determination. ${ }^{14}$ The detection of antigen galactomannan in BAL fluid as a means of establishing early diagnosis seems promising, but this test is not yet widely available. ${ }^{10}$ The selling point of this case resides on negative serology and negative direct examination of BAL, whereas the culture was positive.

Chest CT scan also has limitations: intrahospital transport is often undesirable in hypoxemic patients under mechanical ventilation, observed signs are often obscured by interfering radiologic abnormalities, and furthermore they are not specific for Aspergillus species and can be seen in Mucorales species infections. However, chest CT may be of significant importance in patients with a low suspicion index, as it was in this case. The presence of a halo sign (defined as a macronodule, $\geq 1 \mathrm{~cm}$ in diameter, surrounded by a perimeter of ground-glass opacity) is considered as an early indicator of angioinvasive pulmonary mycosis and was found in $61 \%$ of IPA cases in a large imaging study. ${ }^{8}$ Cavitary lesions are highly suggestive of angioinvasive pulmonary mycosis, especially if an air crescent sign is present in the cavity, but they are less common (respectively, $20 \%$ and $10 \%$ in the same study), and they are considered as markers of later-stage disease. In this case, the presence of a halo sign enables the diagnosis of IPA and not primary aspergilloma or chronic necrotizing aspergilloma, because the latter two may be indistinguishable on noncontrast CT in immunocompetent patients. ${ }^{7}$ Chest CT scan findings observed in the present case were of crucial importance, especially because they were absent on a previous exam. They allowed us to rule out the hypothesis of Aspergillus colonization of the respiratory tract.

\section{Teaching Points}

- Diagnosis of invasive aspergillosis in nonimmunocompromised, critically ill patients remains difficult.

- The ICU patient undergoes relative immune alteration, allowing the development of invasive fungal infections even without immunosuppression criteria.

- IPA is an uncommon cause of ventilator-associated pneumonia in the immunocompetent patient.

- Without histological proof, the diagnosis of IPA is limited to probable or possible IPA when based on a number of arguments (clinical, biological, and radiological).

- Unlike in the immunocompromised patient, serum galactomannan antigen and serum Aspergillus-specific an- tibodies have a limited role in the nonimmunocompromised patient for the diagnosis of IPA. Data on sensitivity and specificity are sparse in this specific population.

- The CT halo sign defined as a macronodule, $\geq 1 \mathrm{~cm}$ in diameter, surrounded by a perimeter of ground-glass opacity, is an early indicator of IPA.

\section{REFERENCES}

1. Stevens DA, Kan VL, Judson MA, Morrison VA, Dummer S, Denning DW, et al. Practice guidelines for diseases caused by Aspergillus; Infectious Diseases Society of America. Clin Infect Dis 2000; 30(4):696-709.

2. De Pauw B, Walsh TJ, Donnelly JP, Stevens DA, Edwards JE, Calandra T, et al. Revised definitions of invasive fungal disease from the European Organization for Research and Treatment of Cancer/ Invasive Fungal Infections Cooperative Group and the National Institute of Allergy and Infectious Diseases Mycoses Study Group (EORTC/MSG) Consensus Group. Clin Infect Dis 2008;46(12):18131821.

3. Meersseman W, Vandecasteele SJ, Wilmer A, Verbeken E, Peetermans WE, Van Wijngaerden E. Invasive aspergillosis in critically ill patients without malignancy. Am J Respir Crit Care Med 2004; 170(6):621-625.

4. Trof RJ, Beishuizen A, Debets-Ossenkopp YJ, Girbes ARJ, Groeneveld ABJ. Management of invasive pulmonary aspergillosis in non-neutropenic critically ill patients. Intensive Care Med 2007; 33(10):1694-1703.

5. Denning DW. Invasive aspergillosis. Clin Infect Dis 1998;26(4): 781-803, quiz 804-805.

6. Garbino J, Fluckiger U, Elzi L, Imhof A, Bille J, Zimmerli S. Survey of aspergillosis in non-neutropenic patients in Swiss teaching hospitals. Clin Microbiol Infect. 2011;17(9):1366-1371.

7. Yoon SH, Park CM, Goo JM, Lee HJ. Pulmonary aspergillosis in immunocompetent patients without air-meniscus sign and underlying lung disease: CT findings and histopathologic features. Acta Radiol 2011;52(7):756-761.

8. Greene RE, Schlamm HT, Oestmann JW, Stark P, Durand C, Lortholary $\mathrm{O}$, et al. Imaging findings in acute invasive pulmonary aspergillosis: clinical significance of the halo sign. Clin Infect Dis 2007; 44(3):373-379.

9. Hope WW, Walsh TJ, Denning DW. Laboratory diagnosis of invasive aspergillosis. Lancet Infect Dis 2005;5(10):609-622.

10. Maertens J, Maertens V, Theunissen K, Meersseman W, Meersseman P, Meers S, et al. Bronchoalveolar lavage fluid galactomannan for the diagnosis of invasive pulmonary aspergillosis in patients with hematologic diseases. Clin Infect Dis 2009;49(11):1688-1693.

11. Klont RR, Mennink-Kersten MA, Verweij PE. Utility of Aspergillus antigen detection in specimens other than serum specimens. Clin Infect Dis 2004;39(10):1467-1474.

12. Mennink-Kersten MA, Donnelly JP, Verweij PE. Detection of circulating galactomannan for the diagnosis and management of invasive aspergillosis. Lancet Infect Dis 2004;4(6):349-357.

13. Vandewoude KH, Blot SI, Depuydt P, Benoit D, Temmerman W, Colardyn F, Vogelaers D. Clinical relevance of Aspergillus isolation from respiratory tract samples in critically ill patients. Crit Care 2006;10(1):R31.

14. Tarrand JJ, Lichterfeld M, Warraich I, Luna M, Han XY, May GS, Kontoyiannis DP. Diagnosis of invasive septate mold infections: a correlation of microbiological culture and histologic or cytologic examination. Am J Clin Pathol 2003;119(6):854-858. 